\title{
Destruction of an advanced malignant tumour by direct electrical current-case report
}

\author{
Chima Oji ${ }^{1 *}$, John $\mathrm{Ani}^{2}$ \\ ${ }^{1}$ Department of Oral and Maxillofacial Surgery, Ebonyi State University Teaching Hospital, Abakaliki, Nigeria; \\ ${ }^{*}$ Corresponding Author: chimaoji@gmail.com \\ ${ }^{2}$ Ntasiobi Specialist Hospital, Enugu, Nigeria
}

Received 6 December 2009; revised 14 May 2010; accepted 24 May 2010.

\begin{abstract}
We carried out a study on the effect of low-level direct current on cancer by using it to treat a woman who had a large malignant squamous cell carcinoma of the sinus cavity. We used a device that produced low-level direct current and passed the current through the tumour via a $4 \times 4 \mathrm{~cm}$ flat aluminium foil and a needle electrode that was insulated along its entire length except for the portion actually inserted into the tumour. The treatment was eight hourly daily and lasted for eight weeks. The therapy resulted in the total remission of the tumour and a feeling of wellness by the patient. This finding implies promising therapeutic potential for the use of direct electrical current as a simple, effective, low cost alternative for the treatment of cancer.
\end{abstract}

Keywords: Destruction; Malignant Tumour; Human Patient; Direct Electrical Current

\section{INTRODUCTION}

Many clinical cases of cancer do not respond to the conventional approaches of surgery, chemotherapy, radiotherapy, hormone therapy and biological therapy. The disadvantages of these cancer treatment modalities include damage to healthy cells and the resulting significant side effects, such as hair loss, fatigue, hormonal changes that may affect fertility and libido, blood clots, flu-like symptoms, and/or complex, risky, expensive surgical procedures. Consequently, a new cancer treatment modality, which uses a device that is minimally invasive, and provides effective treatment without major side effects, is on demand. One of the newer techniques that researchers and clinicians are investigating for its potential role in clinical therapy is electrotherapy, [1-5] Eaton [6] suggested as early as 1776 that electricity might have a role in the treatment of tumours. He reported the case of a patient with a breast tumour whom lightning struck and her tumour retarded. Other investigators of that era used the available electronic techniques to treat tumours with electricity and narrated positive results [6]. Humphrey and Seal [7] reported that a growing foetus or a growing uterine tumour would cause the uterus to be electronegative with respect to the abdominal surface. In the guinea pig and mouse, the tumour is also negative. This supports the many findings that a growing region is electronegative with respect to a slower growing or non-growing region in the same organism, irrespective of plant or animal [7]. The presence of direct current (DC) surface electro-potentials that a microvolt meter can detect and measure, characterizes living tissues [8]. Humphrey and Seal [7], Schauble et al [8] and Habal [9] described the necrosis and retardation of tumour growth in three solid tumour models when they passed low-level direct current through the tumours. According to Weber [10], a cell must replicate its deoxyribonucleic acid (DNA) strand for it to divide. The building blocks of this strand are four bases that are in short supply in a healthy, resting cell. On the other hand, the building blocks of a related molecule, ribonucleic acid (RNA), are always in great abundance because many cellular functions need RNA. When a cell is ready to divide, an enzyme called ribonucleotide reductase (RR), converts building blocks of RNA into those of DNA. The enzyme RR is therefore pivotal for cell growth. The activity of this enzyme is thus tightly linked, much more than that of any other enzyme, to neoplastic transformation and progression. Kulsh [11] promulgated the hypothesis that a novel way of arresting the activity of this all-important enzyme in cell growth lies in the fact that the active site of RR contains a stable tyrosyl free radical, which is essential for its activity [12]. Kulsh [11] surmised that free-floating electrons, which are easily available in the form of direct electric current, could neutralize or destroy such free radicals. Direct current 
electrotherapy should therefore result in inhibition of RR and cessation of malignant cell proliferation. Low-level surface DC electrotherapy would act selectively on cancerous growth since the concentration of the target enzyme RR is exponentially higher in cancerous cells, as compared to healthy quiescent cells [10]. Metastasized cancer should also be treatable by direct current electrotherapy since even in the metastatic state, the biochemical mechanism of cell division involving the enzyme RR remains the same notwithstanding the organ microenvironment.

In view of the fact that both the direction and the spread of electric current can be controlled, thereby limiting the effects to a defined area, we believe that the exploration of this phenomenon holds great promise. It should also be possible to deliver the current to areas of the body that are not accessible to surgery.

A conspicuous and essential point in this article is that the case we present is not a laboratory experiment but a stage IV cancer of the TNM (Tumour, Node, Metastasis) system involving a poor woman in dire condition because of the advanced nature of the disease and because she could not afford the cost of conventional surgery. Furthermore, this is the first time in West Africa that a human patient received direct current therapy.

The aim of this study was to examine the effect of the application of low-level direct current on patients with large malignant oral tumours. We are of the opinion that this novel method of cancer treatment is important in a developing country because it is low-cost, non-toxic, non-invasive, site specific, and easy to administer.

\section{CASE REPORT}

A 60-year-old Nigerian woman presented to our oral and maxillofacial clinic in 2007. She complained of swelling of the right face and the palate that began three years earlier. In addition, she had hearing problems, especially of the right ear. The tumour has steadily been increasing in size (Figure 1). She also complained of blockage of the right nostril, difficulty in breathing and in swallowing, speech impairment and discomfort when chewing. The swellings were tender to touch and the tumour on the palate bled occasionally. The patient's medical history was benign - she denied fever, chills, weight loss, vomiting and nausea. She admitted that she had hitherto patronized unorthodox medical practitioners before a medical doctor referred her to our maxillofacial unit.

Clinical examination showed an elderly, nervous and talkative woman with a facial mass on the right side of the face in moderate respiratory distress. There was also a large swelling (diameter $=4.5 \mathrm{~cm}$ ) on the palate $($ Figure 1). The lesion did not affect the facial nerve. The

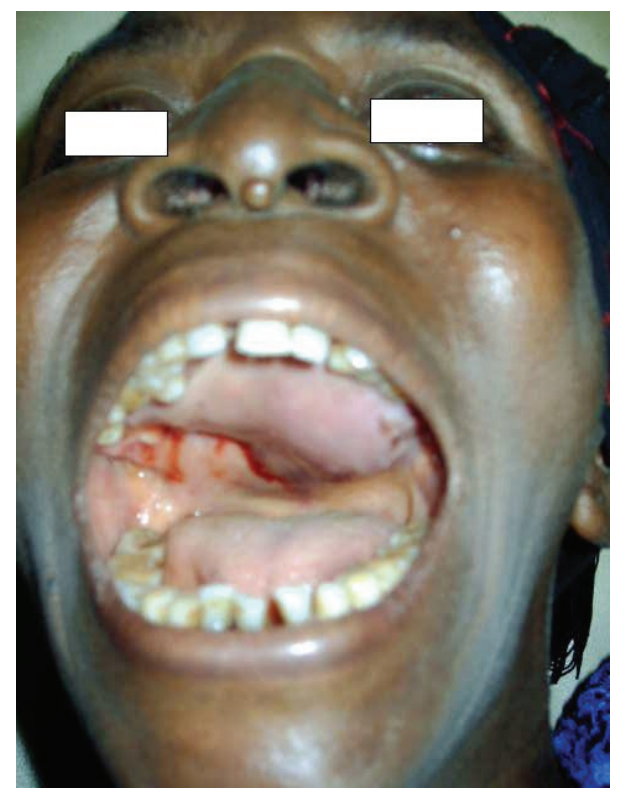

Figure 1. Tumour of the palate before treatment.

radiograph (jug handle or occipitomental view) revealed lytic bone destruction of the right sinus cavity. The biopsy result reported squamous cell carcinoma. We therefore made a diagnosis of squamous cell carcinoma (SCC) in stage IV of the TNM (Tumour, Node, Metastasis) system.

After obtaining ethical clearance from the health institution where we treated the patient; informed consent from her and extra/intraoral photographs, we started treatment using the GEIPE ${ }^{\mathrm{a}}$ device and the following materials: custom electrodes, needle electrodes, electrode gel, and sandpaper strips. The device was battery-powered and provided constant electrical current. In accordance with the treatment protocol of the GEIPE Cancer Treatment, we prepared the skin by removing dead skin cells from the area with very fine sand paper; cleaning with water; drying and finally rubbing conducting gel on the skin surface. We connected the wires first to a passive surface electrode, which was an aluminium foil plate that measured $4 \times 4 \mathrm{~cm}$ (extra oral electrode) and then to an active needle (intra oral electrode). This was a 14-gauge stainless steel injection needle, whose hub was removed. It was insulated with tightfitting silicon tubing that covered the needle except for the $5 \mathrm{~mm}$ tip. The exposed tip had a diameter of 2.25 $\mathrm{mm}$, and a surface area of $0.35 \mathrm{~cm}^{2}$.

We placed the electrodes in such a way that the cancerous tissue fell in the path between them by visualizing a straight line going through the body and through the tumour. We taped the surface electrode to hold it down (Figure 2). After sterilizing the tip of the needle, we inserted it into the tumour so that the exposed tip was 


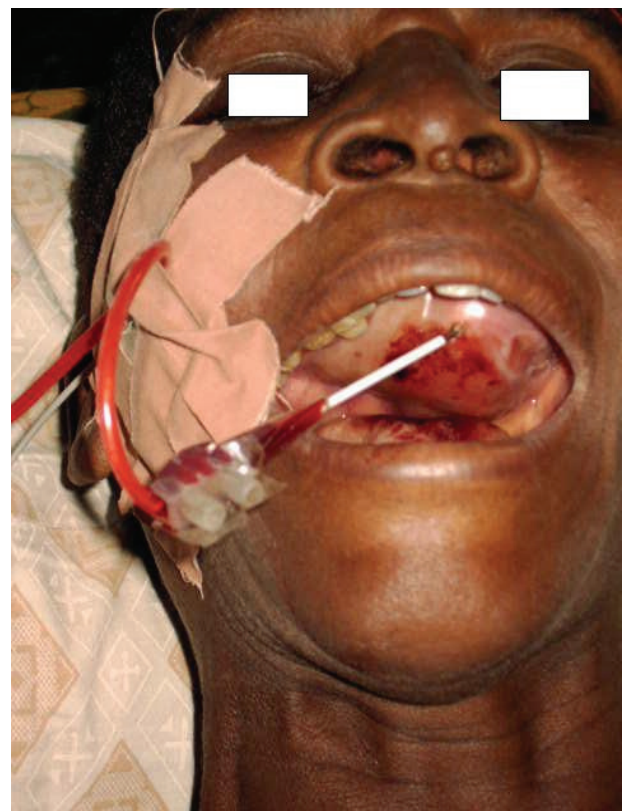

Figure 2. Placement of the electrodes.

within the tumour and switched the device on. Initially, we passed a current of $2 \mathrm{~mA}$ and a voltage of $3 \mathrm{~V}$ through the tumour for one hour. After examining the skin and establishing that there were no adverse reactions, we increased the time of treatment slowly to a maximum of eight hours at a time. The GEIPE device, which was equipped with two ON positions, required that the patient or a health worker activate the first ON switch and then the second ON switch after every five minutes. This was necessary in order to avoid electrolysis and dissolution of electrodes. Since we found this procedure stressful, we consulted an engineering firm, GODIAC ${ }^{\mathrm{b}}$ (Nig.) Ltd that modified the GEIPE device to perform the switching function automatically.

At the end of each treatment session, we gently washed the skin with soap and water and placed a moisturizer on the area. After the first three days of treatment, we observed oedema and discoloration of the lesion in the palate. Seven days later, there was marked necrosis of the lesion. We carefully removed the necrotic tissue and the colour of the palate mucosa normalized in the course of treatment. The extra oral as well as the intra oral tumour flattened after eight weeks of treatment (Figure 3), and the patient's accompanying ailments disappeared. She left the hospital against our advice explaining that she felt well and had neither the money nor the need for further medical attention. This was the reason why we could not execute final radiographic examinations and biopsy. We saw the healthy-looking patient eighteen months later in the marketplace where she sold vegetables. She refused our request for her to come for a check-up.

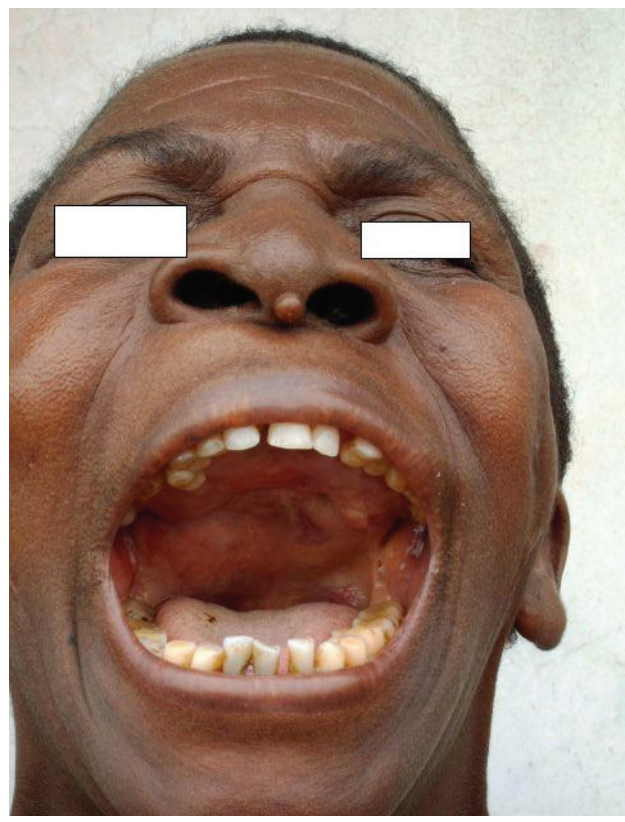

Figure 3. The palate-eight weeks after onset of treatment.

\section{DISCUSSION}

The case that we reported here suggests that direct electric current can destroy an advanced malignant tumour within a relatively short span of time (eight weeks). Many possible mechanisms may account for tumour destruction by direct current. Kulsh [11] postulated the enzyme-mediated mechanism for the first time. He, however, suggested that voltage between 1.2-3 V would be most beneficial for the disabling of RR through free radical interactions. Kulsh [11] was of the opinion that higher voltage for this mechanism would be undesirable because more and more electrons would engage in electrochemical processes leaving less and less electrons free as free radicals, and the concentration of toxic electrochemical species would increase steadily. In our case, we used $3 \mathrm{~V}$.

Yen et al. [13] applied direct current of $400 \mu \mathrm{A}$ at 3 volts for 208.4 minutes (i.e., 5 Coulombs in $5 \mathrm{~mL}$ or 1 Coulomb $/ \mathrm{mL}$ ), to a human cancer cell culture. The $\mathrm{pH}$ at the anode decreased to 4.53 and increased to 10.46 at the cathode. The effect of $\mathrm{pH}$ alteration on cells is thus likely one of the mechanisms of tumour cell destruction.

A 1994 study by Berendson et al. [14], showed that the main reactions at the anode are the formation of oxygen, acidification due to liberated hydrogen ions, and, if platinum is used as anode material, the formation of chloride. At the cathode, hydrogen is formed and hydroxide ions are liberated. Based on calculations, the authors concluded that the liberated hydrogen ions de- 
termine the extents of the locally destroyed zone around the anode and that the destructive effect of chlorine probably occurs in an inner zone close to the anode.

Though Marino et al. [15] employed a current of 2 $\mathrm{mA}$ and a voltage of $3 \mathrm{~V}$ in their experiment, they opined that the $\mathrm{pH}$ changes in the tumour tissue - to the extent that they overcome the body's buffering capacitymight be responsible for the observed effects on tumour growth. They reasoned that the absence of an effect on tumour growth with AC (alternating current) supports this view because in this case, the electrochemical events at each electrode are identical but they do not produce a $\mathrm{pH}$ gradient.

According to Harguindey [16], tumour hyperacidification might activate cytolytic mechanisms through increased activity of lysosomes, resulting in the destruction of tumour tissues. Low $\mathrm{pH}$ also inhibits glycolysis and protein synthesis upon which malignant tissues are dependent.

A positive potential on metal electrodes leads to corrosion of the metal with the release of metal ions from the electrode and possible resultant necrosis and metal toxicity. At the anode, the stainless steel electrode is corroded with the ferrous ions going into solution [8]. In their experiment with mice, Morris et al. [17] detected that DC-induced tumour necrosis was polarity specific. At the anode, it was coagulative and at the cathode, it was ischemic. They surmised that this was further evidence that the mechanism for tumour destruction was electrochemical in nature. Taylor et al. [18] noted that vascular occlusion by thrombosis can be reliably produced by the passage of an appropriate quantity of electrical current.

It must, however, be noted that DC therapy has its limitations, namely, its inability to effect a complete remission in many cases. The percentages of total and partial remissions vary from case to case as evidenced by the works of Turler et al. [19] Kirson et al. [20] and Barbault et al. [21]. Other drawbacks of this therapy its stationary nature (in the case presented here, the patient was in the supine position for eight hours daily) and its duration (again, in our case, the treatment lasted for eight weeks).

All the papers referenced here agree on one issue, namely, that DC destroys tumours. There are, however, diverse views of the way it works. It appears, therefore, that there is yet no clear understanding of the underlying mechanisms of action. Nonetheless, we share the belief of Taylor et al. [18] that there is a great therapeutic potential for the development of this new technology. If further studies can confirm the beneficial effects of direct electrical current on malignant tissues, as we have seen in our study and as shown in other studies, then the application of relatively small amounts of direct electrical current using a variety of purpose-designed delivery electrodes, could produce an innovative low cost treatment alternative for patients with malignant diseases. We urge governments and stakeholders in the health care delivery to encourage and support researchers to continue these studies.

\section{ACKNOWLEDGEMENTS}

We are thankful to Mr. Jay Kulsh, CEO of GEIPE ${ }^{a}$ from whom we bought the GEIPE device. We also acknowledge Mr. Goddy Oku, CEO of GODIAC ${ }^{b}$ (Nig.) Ltd for modifying the GEIPE device.

a. GEIPE Cancer Treatment: GEIPE, P.O. Box 69264 Los Angeles, CA 90069. USA.

b. GODIAC: GODIAC (Nig.) Ltd. No. 2 Grant Street, 400001 Enugu, Nigeria.

\section{REFERENCES}

[1] Emami, B., Nussbaum, G.N., Hahn, N., Piro, A.J., Dntschilo, A. and Quimby, F. (1981) Histopathological study on the effect of hyperthermia on microvasculature. International Journal of Radiation Oncology, Biology and Physics, 7(3), 343-348.

[2] Lindholm, C.C., Kjellen, E., Landberg, T., Nilsson, P. and Persson, B. (1982) Microwave induced hyperthermia and ionizing radiation. Preliminary clinical results. Acta Radiologica Oncology, 21(4), 241-254.

[3] Rand, R.W., Snow, H.D. and Brown, W.J. (1982) Thermomagnetic surgery for cancer. Journal of Surgical Research, 33(3), 177-183.

[4] Urano, M., Rice, L., Epstein, R., Suit, H.D. and Chu, A.M. (1983) Effect of whole body hyperthermia on cell survival, metastases frequency, and host immunity in moderately and weakly immunogenic munne tumors. Cancer Research, 43(3), 1039-1043.

[5] Widder, K.J., Senyei, A.E. and Seas, B. (1982) Experimental methods in cancer therapeutics. Journal of Pharmaceutical Sciences, 71(4), 379-387.

[6] Schechter, D.C. (1979) Flashbacks: Containment of tumors through electricity. PACE, 2(1), 101-114.

[7] Humphrey, C.E. and Seal, E.H. (1959) Biophysical approach toward tumor repression in mice. Science, 130(3372), 388-390.

[8] Schauble, M.K., Habal, M.B. and Gullick, H.D. (1977) Inhibition of experimental tumor growth in hamsters by small direct current. Archives of Pathology \& Laboratory Medicine, 101(6), 294-297.

[9] Habal, M.B. (1980) Effect of applied DC current on experimental tumor growth in rats. Journal of Biomedical Materials Research, 14(6), 789-801.

[10] Weber, G.. (1983) Biochemical strategy of cancer cells and the design of chemotherapy. Cancer Research, 43(8), 3466-3492.

[11] Kulsh, J. (1997) Targeting a key enzyme in cell growth: a novel therapy for cancer. Medical Hypotheses, 49(4), 297-300. 
[12] Miklaĉiĉ, D., Fajgelj, A. and Serša, G. (1994) Tumor treatment by direct electric current: Electrode material deposition. Biosensors, 35(1), 93-97.

[13] Yen, Y, Li, J.R., Zhou, B.S., Rojas, F., Yu, J. and Chou, C.K. (1999) Electrochemical treatment of human kb cells in vitro. Journal of Bioelectromag, 20(1), 34-41.

[14] Berendson, J. and Simonsson, D. (1994) Electrochemical aspects of treatment of tissue with direct current. European Journal of Surgery, 574(Suppl.), 111-115.

[15] Marino, A.A., Morris, D. and Arnold, T. (1986) Electrical treatment of lewis lung carcinoma in mice. Journal of Surgical Research, 41(2), 198-201.

[16] Harguindey, S. (1982) Hydrogen ion dynamics and cancer-An appraisal. Medical and Pediatric Oncology, 10(3), 217-236.

[17] Morris, D., Marino, A.A. and Gonzalez, E. (1992) Electrochemical modification of tumor growth in mice. Journal of Surgical Research, 53(3), 306-309.
[18] Taylor, T.V., Engler, P., Pullan, R.R. and Holt, S. (1994) Ablation of neoplasia by direct current. British Journal of Cancer, 70(2), 342-345.

[19] Turler, A., Schaefer, H., Schaefer, N., Maintz, D., Wagner, M., Qiao, J.C. and Hoelscher, A.H. (2000) Local treatment of hepatic metastases with low-level direct electric current: Experimental results. Journal of Gastroenterology, 35(3), 322-328.

[20] Kirson, E.D., Gurvich, Z., Schneiderman, R., Dekel, E., Itzhaki, A., Wasserman, Y. and Schatzberger, R. (2004) Disruption of cancer cell replication by alternating electric fields. Cancer Research, 64(9), 3288-3285.

[21] Barbault, A., Costa, F.P., Bottger, B., Munden, K.F. and Bonholt, F. (2009) Amplitude-modulated electromagnetic fields for the treatment of cancer. Discovery of tumorspecific frequencies and assessment of a novel therapeutic approach. Journal of Experimental and Clinical Cancer Research, 28(51), 1756-1759. 